A N N A L E S Annales de Bretagne et des Pays de l'Ouest

\title{
Le Han, Marie-Josette(dir.), Saint-Pol-Roux, passeur entre deux mondes
}

\section{Pascal Rannou}

\section{(2) OpenEdition}

1 Journals

Édition électronique

URL : http://journals.openedition.org/abpo/2036

DOI : $10.4000 / a b p o .2036$

ISSN : 2108-6443

\section{Éditeur}

Presses universitaires de Rennes

\section{Édition imprimée}

Date de publication : 30 mai 2011

Pagination : 188-190

ISBN : 978-2-7535-1691-5

ISSN : 0399-0826

\section{Référence électronique}

Pascal Rannou, «Le Han, Marie-Josette(dir.), Saint-Pol-Roux, passeur entre deux mondes », Annales de Bretagne et des Pays de l'Ouest [En ligne], 118-2 | 2011, mis en ligne le 30 juin 2013, consulté le 23 septembre 2020. URL : http://journals.openedition.org/abpo/2036 ; DOI : https://doi.org/10.4000/ abpo. 2036

Ce document a été généré automatiquement le 23 septembre 2020.

(c) Presses universitaires de Rennes 


\title{
Le Han, Marie-Josette(dir.), Saint- Pol-Roux, passeur entre deux mondes
}

\author{
Pascal Rannou
}

\section{RÉFÉRENCE}

LE HAN, Marie-Josette (dir.), Saint-Pol-Roux, passeur entre deux mondes, Rennes, PUR, coll.

«Interférences », 2011, 260 p.

1 Saint-Pol-Roux, le légendaire solitaire de Camaret, reste hélas peu lu de nos jours. Aussi est-ce avec reconnaissance que l'on voit paraitre, sous la direction de Marie-Josette Le Han, les actes du colloque consacré au poète. Dans son avant-propos, M.-J. Le Han exprime l'ambition de ce travail: « cherche[r] à situer cette œuvre complexe dans le champ littéraire, à définir le message esthétique et spirituel qu'elle nous livre aujourd'hui encore. " «Saint-Pol-Roux restait un chercheur solitaire qui assumait le rôle inconfortable de "passeur" entre Symbolisme et modernité ", rappelle M.-J. Le Han. Chaque contributeur va montrer en quoi le mage de Camaret a pu assurer ce rôle de passeur.

2 Jean Roudaut livre à cet ouvrage une introduction particulièrement fervente. Il y montre combien le travail poétique ne se dissocie jamais, chez Saint-Pol-Roux, d'une profonde charge humaine, comme en témoignent les dédicaces, qui sont autant de «marqueurs d'affection ». Le poète considère ses recueils comme des livres d'heures qui scandent la journée, la sienne et celle de ses frères humains. Cette scansion temporelle évoque les «heures canoniales » : le message de l'auteur s'ancre dans le religieux : «Le rôle du poète consiste à faire voir le paradis occulté en notre terre ». Les manuels scolaires, quand ils parlent de Saint-Pol-Roux, le réduisent souvent à un faiseur d'assonances et de métaphores. J. Roudaut dénonce cette lecture sommaire, 
voyant dans ces pratiques non de la "préciosité » mais « une conception évolutive du monde ».

3 Georges Reynaud analyse ensuite "les années de formation de Saint-Pol-Roux ", de 1861 à 1882, suivant le parcours familial, scolaire et militaire de l'auteur. « On notera dans cette œuvre l'abondance de termes en provençal, langue qui a baigné l'enfance du poète » écrit G. Reynaud. Saint-Pol-Roux est donc aussi passeur entre deux langues, trois même, puisque la contribution suivante, due à Marcel Burel, a pour objet «Breton et bretonnismes dans les poèmes roscanvélites de Saint-Pol-Roux ». Avant de s'établir à Camaret, le poète a vécu quelques années au hameau de Lanvernazal, en Roscanvel, dont il a célébré la vie locale avec tendresse. Le poème intitulé « Coupe de goémon en Roscanvel» est une fresque épique qui en magnifie les protagonistes. "Quant aux bretonnismes que Saint-Pol-Roux accueille [...], ils enchantent l'oreille par leur fraîcheur et leur saveur poétique", écrit M. Burel. "Entre Iroise et émeraude", Dominique Bodin évoque ensuite la relation qui unit deux « solitaires » de la poésie que furent Saint-Pol-Roux et Théophile Briant. De Camaret à Paramé, l'un comme l'autre furent inspirés par la mer, et leur fin brutale n'est pas le seul point commun de deux existences vouées à « une haute idée de la poésie et du poète ».

Trois articles ont pour sujet la correspondance de Saint-Pol-Roux. Jean-Louis Debauve en dresse tout d'abord l'inventaire raisonné. Puis Jean-André Le Gall se penche sur celle que Saint-Pol-Roux a entretenue avec Victor Segalen. Il détruit la légende qui dépeint souvent ces deux hommes comme deux frères en poésie : Segalen n'a-t-il pas offert à l'ermite de Camaret les bois de la "Maison du Jouir », qui ornaient la maison de Gauguin? Certes, mais Saint-Pol-Roux, toujours à court d'argent, entreprit de les revendre, alors que Segalen les lui réclamait pour illustrer la correspondance de Gauguin. Les deux hommes furent, en outre, épris chacun d'une conception radicalement différente de l'exotisme: spatial et nomade, chez le grand voyageur que fut Segalen; temporel et sédentaire, pour le menhir enraciné que fut Saint-Pol-Roux : " sauf à se convertir à l'exotisme de l'autre, ces deux solitaires ne pouvaient demeurer solidaires bien longtemps » conclut plaisamment J.-A. Le Gall. Nicolas Tocquer dresse lui aussi le bilan d'une autre correspondance, celle qui lia cette fois le Breton d'adoption à son autre "pays", Jean Royère, l'influent directeur de La Phalange. On retrouve ici le visage peu glorieux que Saint-Pol-Roux montra dans l'affaire des bois de Gauguin. Le poète voudrait que Royère favorise sa nomination au Musée de Pau, voire son obtention de la Légion d'honneur... Loin des réseaux influents, le solitaire de Camaret vit pauvrement, et confie à Royère la tâche de vendre des pièces de sa collection d'objets d'art africain.

René Mabin envisage "Saint-Pol-Roux, frère des peintres (de Gauguin à Jim Sévellec) ». Comme beaucoup de poètes, le Magnifique ne dissocie pas son art des autres formes d'expression. La peinture lui inspire cet étonnant poème pictural intitulé «L'œil goinfre ", qui le rend proche des Impressionnistes. Saint-Pol-Roux aura su reconnaître les peintres qui compteront pour les générations ultérieures, notamment Gauguin et Van Gogh. Leurs figures de victimes du pharisaïsme ambiant ne pouvaient qu'épouser sa démarche. Dans son étude sur « Saint-Pol-Roux et le primitivisme », Françoise Daniel rappelle d'ailleurs combien Saint-Pol-Roux aurait pu faire siennes les paroles de Gauguin parcourant la Bretagne: «J'aime la Bretagne, j'y trouve le sauvage, le primitif.» 
6 Abordant La Dame à la faulx, Jean-Louis Meunier se demande s'il faut en " lire » ou en « jouer les didascalies. » Ce drame est, en effet, avant tout « du théâtre à lire ». J.-L. Meunier montre que les didascalies y illustrent la grande ambition de l'auteur « de parachever un drame total dans une perspective wagnérienne qui lui était contemporaine ", de "donne[r] à voir et à entendre ce que les dramaturges qui l'avaient précédé donnaient à dire par le récit [...] ou par le monologue ». C'est dire qu'on est déjà proche d'une conception de la didascalie qui, loin d'être purement informative, a un statut littéraire et dramatique propre.

7 Amateur d'art dans tous les sens du terme, Saint-Pol-Roux l'est donc aussi de musique, et c'est sur son « univers sonore » que se penche Patrick Besnier. Il montre que SaintPol-Roux a toujours voulu associer la musique à son théâtre, sollicitant même, mais en vain, Debussy et Ravel quand La Dame à la faulx fut jouée en 1911. P. Besnier montre que l'influence de Wagner sur Saint-Pol-Roux reste incontournable, mais que celui-ci paraît subordonner la musique à l'écriture, puisqu'« il va jusqu'à parler de la musique comme “dégénérescence du Verbe" ". P. Besnier nous fait découvrir un Saint-Pol-Roux surprenant, dont les indications sonores annoncent bruitisme et futurisme. Le poète entretient avec le quatrième art une relation conflictuelle, où la fascination le dispute à l'incompréhension.

8 «Fils du soleil», Saint-Pol-Roux fut aussi un grand lecteur de Rimbaud, comme le montre Jean-Luc Pestel: mais du jeune poète révolté, le solitaire retient surtout le message "johannique » et prophétique, celui du Voyant, quand l'habitait encore une puissance messianique, avant le temps du silence et des désillusions. Saint-Pol-Roux rend à Rimbaud un hommage vibrant dans Les Traditions de l'avenir et s'inspire des Illuminations, signale J.-L. Pestel, dont l'article montre tout ce que Saint-Pol-Roux doit à son aîné, et ce qu'il retient de son héritage.

9 Julien Schuh et Jacques Goorma se penchent tous deux sur la notion délicate d "idéoréalisme ", par laquelle Saint-Pol-Roux a défini sa poésie. J. Schuh montre bien que le sens de ce mot n'a pas toujours été fixe dans l'esprit de Saint-Pol-Roux. Si l'idéoréalisme représente au début pour lui « une critique des points de vue purement idéaliste (le Symbolisme) ou matérialiste (le Naturalisme), et d'une proposition de voie médiane ", Saint-Pol-Roux enrichira sans cesse cette notion, dont J. Schuh montre bien la complexité. En proposant un rigoureux «schéma de l'idéoréalisme », J. Goorma montre que cette notion exprime " la poésie comme force d'union et d'émancipation »: union des contraires (chair et esprit, vie et mort, « fixe et volatil»...), essor vers la vérité divine.

D. Millet-Gérard se livre dans «Mystère des êtres, mystère des choses, mystère du verbe " à une intéressante étude comparée entre trois poètes: Saint-Pol-Roux et ses cadets Francis Jammes et Armand Godoy. L'étude fine du poème «Chapelle de hameau ", du premier, montre « chez nos trois poètes : un accroissement de la lisibilité de Saint-Pol-Roux à Jammes puis à Godoy ». Plus complexe, la poétique de Saint-PolRoux est celle d'un passeur entre le Nature et l'Idée, et son " autre monde » ne se réduit pas à l'" eschatologie chrétienne " de ses deux confrères. Mikaël Lugan consacre une riche étude aux liens que le barde de Camaret noua avec le Surréalisme. "Idéoréalisme et Surréalisme s'accordent d'abord sur le constat de l'insuffisance du réel », mais aussi sur une conception plus radicale de la création métaphorique. Saint-Pol-Roux annonce les recherches surréalistes, notamment celle de l'écriture automatique. Mais le mysticisme du barde breton s'accommodait sans doute mal de l'éthique surréaliste. 
11 Jean-Michel Kervran aborde les "notions d'esthétique et d'éthique chez Saint-PolRoux » et son rapport à la spiritualité. Il montre comment Saint-Pol-Roux, disciple de Plotin, rénove les conceptions esthétiques de son époque. Saint-Pol-Roux «fait feu de tout mot ». Son écriture foisonne. Il emprunte aussi des mots au wallon, et use d'un « lexique volontairement archaïque ». Mais cette esthétique véhicule une éthique : « La quête poétique est indissociable de la quête philosophique voire métaphysique. » Odile Hamot nous brosse d'ailleurs le « Portrait du poète en Ezéchiel : à l'image du prophète Ezéchiel, [il] se voit investi de la plus haute mission : voir le mystère et transfigurer le monde par sa parole ». M.-J. Le Han clôt ce copieux volume par une subtile analyse du poème "Le châtelain et le paysan", qui définit son auteur comme "pèlerin de la charité »: «Il semble avoir renoncé à son individualité pour devenir l'interprète d'une humanité silencieuse et courageuse. » S'il en fallait une preuve, M.-J. Le Han rappelle à bon escient que, dès 1933, dans sa Supplique au Christ dédiée à Einstein, Saint-Pol-Roux plaignait le sort des Juifs dans l'Allemagne nazie.

12 Les actes de ce colloque ont donc permis à de fervents lecteurs de Saint-Pol-Roux de s'exprimer en déchiffrant, toujours avec une grande clarté, le message du poète. On peut regretter qu'on y parle souvent plus autour de l'œuvre qu'on n'y analyse l'œuvre en elle-même, mais les textes réunis ici en appellent évidemment d'autres. 